\title{
The examination of sleep quality for frontline healthcare workers during the outbreak of COVID-19
}

\author{
Haitham Jahrami ${ }^{1,2}$ (D) Ahmed S. BaHammam ${ }^{3,4}$. Haifa AlGahtani ${ }^{2} \cdot$ Ahmed Ebrahim $^{1} \cdot$ MoezAllslam Faris $^{5}$. \\ Kawthar AlEid ${ }^{1} \cdot$ Zahra Saif $^{1} \cdot$ Eman Haji $^{1} \cdot$ Ali Dhahi $^{1} \cdot$ Hussain Marzooq ${ }^{1} \cdot$ Suad Hubail $^{1} \cdot$ Zainab Hasan $^{1}$
}

Received: 23 April 2020 / Revised: 4 June 2020 / Accepted: 17 June 2020 / Published online: 26 June 2020

(C) Springer Nature Switzerland AG 2020

\begin{abstract}
Purpose Few studies have addressed the sleep disturbances of healthcare workers during crisis events of public health. This study aimed to examine the sleep quality of frontline healthcare workers (FLHCW) in Bahrain during the COVID-19 pandemic, and compare it with the sleep quality of non-frontline healthcare workers (NFLHCW).

Methods Healthcare workers $(n=280)$ from multiple facilities belonging to the Ministry of Health, Bahrain, were invited to participate in this cross-sectional study. An online questionnaire, including socio-demographics, the Pittsburgh Sleep Quality Index (PSQI), and the Perceived Stress Scale (PSS), was used to evaluate sleep disturbances and stress levels of healthcare workers. Poor sleep quality was defined as PSQI $\geq 5$ and moderate-severe stress as PSS $\geq 14$. Descriptive statistics were used to compare the scores of FLHCW and NFLHCW. Univariate and multivariate binary logistic regressions were used to identify predictors of poor sleep quality, moderate-severe stress, and the combined problem of poor sleep quality and moderate-severe stress.

Results A total of 257 participants (129 FLHCW and 128 NFLHCW) provided usable responses. The overall PSQI and PSS scores were $7.0 \pm 3.3$ and $20.2 \pm 7.1$, respectively. The FLHCW scored higher in the PSQI and PSS compared with the NFLHCW; however, the differences in the PSQI and PSS scores were not statistically significant. For the FLHCW, 75\% were poor sleepers, $85 \%$ had moderate-severe stress, and $61 \%$ had both poor sleep quality and moderate-severe stress. For the NFLHCW, $76 \%$ were poor sleepers, $84 \%$ had moderate-severe stress, and $62 \%$ had both poor sleep quality and moderate-severe stress. Female sex and professional background were the predictors of poor sleep quality and stress.

Conclusions Poor sleep quality and stress are common during the COVID-19 crisis. Approximately, $60 \%$ of both FLHCW and NFLHCW have poor sleep quality combined with moderate-severe stress.
\end{abstract}

Keywords Bahrain · Coronavirus disease $\cdot$ Pandemic $\cdot$ Perceived stress scale $\cdot$ Pittsburgh Sleep Quality Index · SARS-CoV-2

Haitham Jahrami

hjahrami@health.gov.bh

Ministry of Health, Manama, Kingdom of Bahrain

2 College of Medicine and Medical Sciences, Arabian Gulf University, Manama, Kingdom of Bahrain

3 Department of Medicine, College of Medicine, University Sleep Disorders Center, King Saud University, Box 225503, Riyadh 11324, Saudi Arabia

4 The Strategic Technologies Program of the National Plan for Sciences and Technology and Innovation in the Kingdom of Saudi Arabia, Riyadh, Saudi Arabia

5 Department of Clinical Nutrition and Dietetics, College of Health Sciences/Research Institute of Medical and Health Sciences (RIMHS), University of Sharjah, Sharjah, United Arab Emirates

\section{Introduction}

Coronaviruses are a group of enveloped non-segmented positive-sense RNA viruses; they are broadly distributed in humans and other mammals $[1,2]$. Most human coronavirus infections are mild; however, some cases develop multi-organ infections, with the respiratory system being the most susceptible [3]. In December 2019, a collection of patients with pneumonia of unknown cause were reported in the city of Wuhan, Hubei Province, China; a novel coronavirus was recognized with human-to-human transmission mode [3, 4]. The virus name is SARS-COV-2, which causes the coronavirus disease 2019 (COVID-19) as named by the World Health Organization (WHO) [5]. To date (June 2, 2020), about 6,400,000 confirmed cases and more than 378,000 deaths attributable to this disease have been reported worldwide [6]. 
Outbreaks of infectious diseases, including the current COVID-19, are associated with major psychological distress and significant symptoms of mental illness [7-9]. Healthcare workers may experience symptoms of sleep problems, anxiety, depression, and stress when facing a major incidence of public health threat [10-12].

Subjective sleep quality is defined by the satisfaction of one's overall sleep experience, including aspects of sleep initiation, sleep maintenance, sleep quantity, and refreshment upon awakening [13]. Objective sleep quality consists of the total duration of sleep, the architecture of sleep, the amount of wake during sleep episode, and the frequency of awakenings across the night [13]. Often, sleep quality measurement refers both to the subjective perception of sleep measured using a questionnaire, or a clinical scale, and to objective measures derived from lab recordings, most often from polysomnographic (PSG) recordings [14].

In the general population, poor sleep quality has been estimated to be between 9 and 45\% [15]. In general, research on healthcare workers revealed that approximately $50 \%$ of physicians have poor sleep quality [16-18]. Professionals in the healthcare industry are constantly under stress; deal with sickness, suffering, and death; and have an irregular work schedule and frequent shifts, which may all adversely impact their sleep [19-24]. The outbreak of COVID-19 in Bahrain remains to be a serious challenge for healthcare workers. They are under constant risk of contracting the virus and be infected with the disease from their patients. They are also under great stress of keeping themselves and their family healthy; these may all lead to acute sleep problems, including poor sleep quality and short sleep duration [25].

Very few studies have concentrated on the sleep quality of healthcare workers during pandemic events, especially in the outbreak of COVID-19. Therefore, the current study was designed to examine the sleep quality of healthcare workers in the confrontation with COVID-19, and to make a comparison between healthcare workers in the frontline and those not in the frontline. Frontline healthcare workers are defined in our research by those professionals in organizations dedicated to the assessment, quarantine, isolation, and treatment of established COVID-19 cases. We hypothesized that healthcare workers in frontline would have a poorer sleep quality profile compared with colleagues not directly engaged in the front line.

\section{Materials and methods}

This cross-sectional study was planned and completed using the principles of the guidelines of the Strengthening the Reporting of Observational Studies in Epidemiology [26].

\section{Ethical consideration}

The Research Ethics Committee (REC) of the Secondary Healthcare Research Committee/Ministry of Health, Bahrain, approved the research (SHCRC/06/04/2020), and data collection was started after receiving the approval. Electronic informed consent was sought and obtained from each participant. Participation was voluntary, with no monetary or non-monetary incentives given, and the participant was permitted to withdraw at any time. The study was conducted following the Declaration of Helsinki for human research.

\section{Setting and participants}

The study was conducted using an online survey in April 2020. Frontline healthcare workers $(n=138)$ were recruited using purposive sampling from two COVID-19 locations belonging to the Ministry of Health, Kingdom of Bahrain. Frontline healthcare workers (FLHCW) were defined as those working in the isolation/quarantine units, fever clinics, nasal swab clinics, and those looking after patients with a confirmed diagnosis of COVID-19.

Non-frontline healthcare workers (NFLHCW) $(n=145)$ were selected through a convenience non-probability sample using the acquaintances of the research team. Participants from both groups were invited to take part in the survey via an online web link sent using social network platforms such as WhatsApp messenger offered by WhatsApp Inc. When the participants clicked on the link, they were taken to an electronic Google Form, starting with a description for the study objectives, consent of participation, and other ethical points. Google Form saves each filled questionnaire in the principal investigator's Google drive.

The inclusion criteria for this study were as follows: (1) participants with more than 6 months of service in Ministry of Health, Bahrain; (2) professional healthcare workers including medical doctors, registered nurses, and state registered allied staff; (3) agreed to participate in the survey. The exclusion criteria were as follows: (1) healthcare workers who were not actively on duty at the time of the survey due to any leave of absence; (2) significantly incomplete data of the online questionnaires.

\section{Tools and techniques}

An English language, self-administered questionnaire was used to collect the data, as the professional communication language of Medicine in Bahrain is English. The questionnaire consisted of structured, closed-ended questions. There were no open-ended or continuing questions, making the questionnaire simple and quick to answer. Based on a pilot test, the research team estimated that it would take each participant around 7 to $10 \mathrm{~min}$ to complete the questionnaire. The 
questionnaire was divided into three domains: socio-demographics, the Pittsburgh Sleep Quality Index (PSQI) [27], and the Perceived Stress Scale (PSS) [28].

\section{Variables}

As described above, the survey collected data on several socio-demographic variables. These included basic data to preserve the anonymity of the participants, including age, sex, marital status, professional background, and type of facility affiliated with (to distinguish between FLHCW and NFLHCW).

The PSQI is a well-known self-reported tool that assesses sleep quality over 1 month. The measure consists of 19 individual items, generating seven components that produce one global score, and takes 5-10 min to complete [27]. The seven components include (1) subjective sleep quality, (2) sleep latency, (3) sleep duration, (4) habitual sleep efficiency, (5) sleep disturbances, (6) use of sleep-promoting medications, and (7) daytime dysfunction due to sleepiness [27]. The PSQI has been used in many settings and with multiple populations [29]. A recent meta-analysis confirmed that the PSQI has superior content validity, construct validity, and discriminant validity [30]. It has a sensitivity of $89.6 \%$ and specificity of $86.5 \%$ for identifying cases with a sleep disorder, using a cutoff score of 5 points [31]. A global PSQI score $\geq 5$ is suggestive of poor sleep quality [27].

The PSS, also known as Cohen's PSS, is the most widely used psychological instrument for measuring the perception of stress [32]. The tool measures the degree to which situations in one's life are appraised as stressful originally using a 14-item scale [33], which was later reduced to 10-item scale [28]. Individual scores on the PSS can range from 0 to 40, with higher scores indicating higher perceived stress. Scores ranging from 0 to 13 would be considered low stress, scores ranging from 14 to 26 would be considered moderate stress, while scores ranging from 27 to 40 would be considered high perceived stress [26]. A review of the psychometric evidence of the PSS concluded that PSS is an easy-to-use tool with established acceptable psychometric properties [34]. The PSS was deemed appropriate in this research to be used as a complementary measure to examine the perceived stress of healthcare workers. This allows making a correlation between sleep quality and levels of stress especially during emergency and unusual times.

\section{Statistical analysis}

The data were analyzed using the STATA 16.1 (version 16, 2019, College Station, TX; StataCorp LLC) [35]. Descriptive statistics were used to summarize participants' sociodemographic factors. The arithmetic mean $(\overline{\mathrm{x}})$ and standard deviation (SD) were reported for continuous variables, and counts and percentages were reported for categorical variables. To compare the two groups, the independent sample $t$ test was used for continuous variables, and Chi-square $\chi^{2}$ was used for categorical variables. The Pearson product-moment correlation coefficient $r$ was used to measure the strength and direction of association that exist PSQI and PSS on a continuous scale.

To assess the predictors of poor sleep quality, moderatesevere stress, or the combination of both poor sleep quality and stress, a univariate logistic regression model was applied in a preliminary analysis where one explanatory variable was tested in the model at a time. Tested variables included demographics, age, sex, marital status, professional background, and being a frontline or non-frontline. All variables then entered in a multivariate logistic regression to define the independent predictors of high risk for poor sleep quality, stress, or both. Odds ratios were computed with $95 \%$ confidence intervals $(95 \% \mathrm{CI})$. Poor sleep quality was defined as PSQI $\geq 5$ and moderate-severe stress as PSS $\geq 14$.

\section{Results}

\section{Participants' socio-demographic characteristics}

A total of 264 of the 280 approached healthcare workers responded to the study, with a response rate of $94 \%$. Seven forms contained significant missing data and therefore were excluded from the analyses leaving us with 257 cases (129 in FLHCW and 128 in NFLHCW). The two groups were very homogenous in their socio-demographic characteristics. The overall mean age was $40.2 \pm 9.7$ years, about $70 \%$ were females, and approximately $90 \%$ were married. Staff nurses represented $50 \%$ of the sample, medical doctors came second with about $30 \%$, and finally, allied healthcare professionals were about $20 \%$. Table 1 provides the socio-demographic characteristics of the study participants and a comparison between FLHCW and NFLHCW.

Participants' sleep quality and perceived stress were measured using the PSQI and the PSS, respectively. Data on the PSQI and PSS scores are presented visually in Fig. 1 and Table 2. The mean PSQI for the entire sample was $7.0 \pm 3.3$, and FLHCW was slightly higher compared with noneFLHCW counterparts; however, no statistically significant difference was detected. The prevalence of poor sleep quality according to the utilized sleep index was $75 \%$, using the standard cutoff of 5 points. The prevalence of poor sleep quality drops to $64.6 \%$ and $51.2 \%$ using cutoffs of 6 points and 7 points, respectively. No statistical difference was obtained when comparing males with females $(P=0.9)$. Single participants scored a mean PSQI score of $7.9 \pm 0.6$ compared with married participants who scored $6.9 \pm 0.2$; this difference did not reach statistical significance. 
Table 1 Socio-demographic characteristics of the study participants

\begin{tabular}{|c|c|c|c|c|}
\hline Variable & Overall $N=257$ & FLHCW $N=129$ & NFLHCW $N=128$ & $P$ Value \\
\hline \multicolumn{5}{|l|}{ Sex } \\
\hline Male & $77(30.0 \%)$ & $40(31.0 \%)$ & $37(28.9 \%)$ & \multirow[t]{2}{*}{0.7} \\
\hline Female & $180(70.0 \%)$ & $89(69.0 \%)$ & $91(71.1 \%)$ & \\
\hline \multicolumn{5}{|l|}{ Marital status } \\
\hline Single & $28(10.9 \%)$ & $16(12.4 \%)$ & $12(9.4 \%$ & \multirow[t]{2}{*}{0.5} \\
\hline Married & $229(89.1 \%)$ & $113(87.6 \%)$ & $116(90.6 \%)$ & \\
\hline \multicolumn{5}{|l|}{ Professional background } \\
\hline Medical doctor & $80(31.1 \%)$ & $43(33.3 \%)$ & $25(19.5 \%)$ & \multirow[t]{3}{*}{0.2} \\
\hline Registered nurse & $119(46.3 \%)$ & $53(41.1 \%)$ & $66(51.6 \%)$ & \\
\hline Allied healthcare professionals & $58(22.6 \%)$ & $33(25.6 \%)$ & $37(28.9 \%)$ & \\
\hline Age (year) & $40.2 \pm 9.7$ & $39.7 \pm 9.9$ & $40.5 \pm 9.5$ & 0.7 \\
\hline
\end{tabular}

$F L H C W$, frontline healthcare workers; $N F L H C W$, non-frontline healthcare workers
The PSQI score of medical doctors was $6.6 \pm 3.3$, staff nurses scored $7.0 \pm 3.2$, and other healthcare professionals scored 7.8 \pm 3.4 . The difference in PSQI scores between the different professional backgrounds was not statistically significant $(P=0.11)$. Close inspection of the seven components of the PSQI revealed that the FLHCW group had lower subjective sleep quality, longer sleep latency, longer sleep duration, similar sleep efficacy, more sleep disturbances, slightly more use of sleep-promoting medications, and slightly more daytime dysfunction compared with the NFLHCW group.

The mean PSS score for the entire sample was 20.2 \pm 7.1 , and the score of the FLHCW group was slightly higher compared with the NFLHCW counterparts; however, no statistically significant difference was detected. Generally, females scored a mean score of $20.7 \pm 0.5$, which is higher than males $19.2 \pm 0.9$; however, the difference was not statistically significant. Single participants scored a mean score of $20.6 \pm 1.2$ compared with married participants $20.2 \pm 0.5$. Medical doctors and staff nurses and other healthcare professionals also appeared to have a similar PSS profile with values of $20.3 \pm 7.6,20.1 \pm 7.1$, and $20.4 \pm 6.3$, respectively. The difference in PSS scores between the different professional backgrounds was not statistically significant $(P=0.96)$.

Table 3 presents the strength of the association between sleep quality and perceived stress. The results of several tests indicated that the PSQI scores were not associated with the PSS scores. Pearson productmoment correlation coefficient indicated a weak positive association between the two variables, but this was not statistically significant at $P<0.05$.

In a subsequent analysis, we categorized the participants into two categories based on the PSQI score and the PSS score severity. The results showered that 191 participants $(75.2 \%$ of the total sample) were of poor sleep quality (a global PSQI score $\geq 5$ ). On the other hand, 216 (84\%) were experiencing moderate-severe stress (a PSS score of 27-40). A total of 157
Fig. 1 The distribution of the PSQI and PSS scores among the healthcare workers

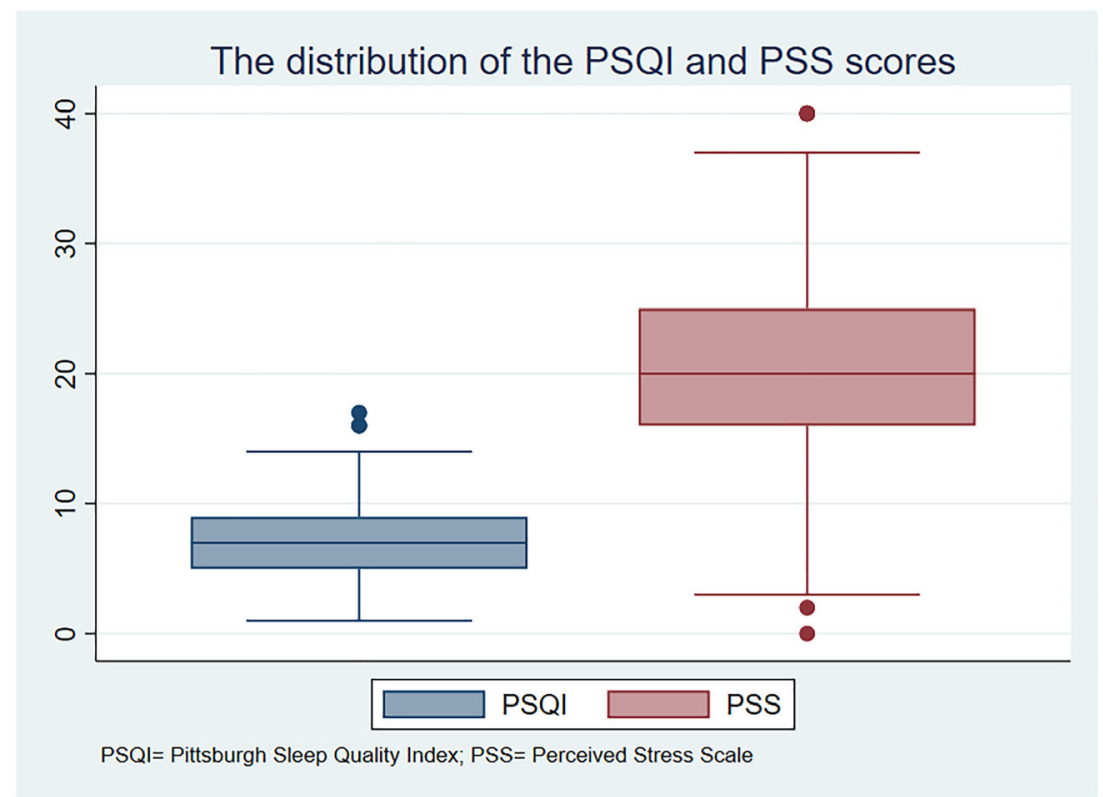


Table 2 Findings of the PSQI and PSS scores

\begin{tabular}{|c|c|c|c|c|}
\hline Variable & Overall $(N=257)$ & FLHCW $(N=129)$ & NFLHCW $(N=128)$ & $P$ value \\
\hline PSQI (C1) subjective sleep quality & $1.2 \pm 0.8$ & $1.3 \pm 0.8$ & $1.1 \pm 0.7$ & 0.1 \\
\hline PSQI (C2) sleep latency & $1.6 \pm 1.0$ & $1.4 \pm 1.0$ & $1.3 \pm 1.0$ & 0.7 \\
\hline PSQI (C3) sleep duration & $1.0 \pm 1.0$ & $1.0 \pm 1.0$ & $1.0 \pm 1.1$ & 0.9 \\
\hline PSQI (C4) habitual sleep efficiency & $0.7 \pm 1.1$ & $0.7 \pm 1.0$ & $0.8 \pm 1.0$ & 0.5 \\
\hline PSQI (C5) sleep disturbances & $1.3 \pm 0.6$ & $1.4 \pm 0.6$ & $1.2 \pm 0.6$ & $0.02 *$ \\
\hline PSQI (C6) use of sleep-promoting medications & $0.4 \pm 0.7$ & $0.4 \pm 0.7$ & $0.3 \pm 0.7$ & 0.4 \\
\hline PSQI (C7) daytime dysfunction & $1.1 \pm 0.8$ & $1.2 \pm 0.8$ & $1.1 \pm 0.8$ & 0.3 \\
\hline Global PSQI & $7.0 \pm 3.3$ & $7.1 \pm 3.5$ & $6.9 \pm 3.1$ & 0.4 \\
\hline Prevalence of poor sleep quality ( $\geq 5$ points) & $191(75.2 \%)$ & $94(74.6 \%)$ & $97(75.8 \%)$ & 0.3 \\
\hline PSS & $20.2 \pm 7.1$ & $20.7 \pm 7.0$ & $19.9 \pm 7.1$ & 0.3 \\
\hline $\begin{array}{l}\text { Prevalence of low stress ( } 0-13 \text { points) } \\
\text { Prevalence of moderate stress (14-26 points) }\end{array}$ & $\begin{array}{l}41(15.9 \%) \\
172(66.9 \%)\end{array}$ & $\begin{array}{l}20(15.5 \%) \\
81(62.8 \%)\end{array}$ & $\begin{array}{l}21(16.4 \%) \\
91(71.1 \%)\end{array}$ & 0.1 \\
\hline Prevalence of severe stress (27-40 points) & $44(17.1 \%)$ & $28(21.7 \%)$ & $16(12.5 \%)$ & \\
\hline
\end{tabular}

The italics only to highlight global or overall score

FLHCW, frontline healthcare workers; NFLHCW, non-frontline healthcare workers; PSQI, Pittsburgh Sleep Quality Index; PSS, Perceived Stress Scale

(61.1\%) had both poor sleep quality and experiencing moderate-severe stress.

Table 4 provides the results of the univariate and multivariate binary logistic regression models for predicting poor sleep quality, moderate-severe perceive stress, and the combination of both among healthcare workers. No predictors could be identified for poor sleep quality. For moderate-severe stress, sex was the only independent predictor. Female sex had an OR 2.0 (95\% CI 1.1-4.0), $P=0.04$. The independent predictors for combined poor sleep quality and moderate-severe stress were female sex (OR 2.0 [95\% CI 1.1-3.5], $P=0.01$ ) and being a health-allied (non-physician) (OR 0.7 [95\% CI 0.5-1.1], $P=0.05$ ).

\section{Discussion}

This is the first study to examine the sleep quality and perceived stress among frontline medical staff in the Arab world and among the first in the world using the PSQI and the PSS during the COVID-19 pandemic. A major finding of this study is that FLHCW do not statistically differ from NFLHCW in sleep quality or stress. The mean global PSQI of the study group was 7.1 indicating poor sleep quality, and the mean PSS score was 20.3, which reflects moderate stress. The findings translate to that $75 \%$ of healthcare workers had poor sleep quality, and $84 \%$ had moderate-severe stress. A total of $61 \%$ of the studied participants had both poor sleep quality and moderate-severe stress. There was no significant linear association between PSQI and PSS scores. Female sex $(\mathrm{OR}=2.0,95 \% \mathrm{CI}=1.1-3.5, P=0.01)$ and professional background $(\mathrm{OR}=0.7,95 \% \mathrm{CI}=0.5-1.1, P=0.05)$ were predictors for poor sleep quality and moderate-severe stress using a multivariate model.
This prevalence rate of poor sleep quality was higher than most of the studies that assessed sleep quality among healthcare workers. For example, in a sample of US doctors working in the emergency room, Machi et al. reported that the prevalence of poor sleep quality was $31 \%$ [36]. Another study in Pakistan reported that poor sleep quality was $37 \%$ among physicians [37]. A recent study in the region, in Saudi Arabia, reported that $51 \%$ of primary care physicians had poor sleep quality [38]. The higher prevalence of poor sleep quality in this study may be related to concerns related to the contagious nature of COVID-19. This is supported by a recent study, in China, of 180 medical staff who treated patients with COVID-19 infection that reported a higher mean PSQI score of $8.6 \pm 4.6$ comparable with our results [39]. Another recent study from China on insomnia among frontline health professionals (using the Insomnia Severity Index) reported that $36 \%$ of those healthcare professionals had reported sleep disturbances [40]. Another

Table 3 The association between sleep quality (PSQI) and perceived stress (PSS)

\begin{tabular}{|c|c|c|c|}
\hline & Overall & FLHCW & NFLHCW \\
\hline \multicolumn{4}{|c|}{ PSQI continuous variable (a) } \\
\hline PSS & $r=0.1, P=0.3$ & $r=0.1, P=0.4$ & $r=0.04, P=0.7$ \\
\hline \multicolumn{4}{|c|}{ PSQI categorical variable (b) } \\
\hline PSS & $X^{2}=2.8, P=0.3$ & $X^{2}=1.4, P=0.5$ & $X^{2}=1.8, P=0.4$ \\
\hline
\end{tabular}

FLHCW, frontline healthcare workers; $N F L H C W$, non-frontline healthcare workers; PSQI, Pittsburgh Sleep Quality Index; PSS, Perceived Stress Scale

* Significant at 0.05

a Pearson product-moment correlation coefficient $r$

b Pearson chi-square $\left(\chi^{2}\right)$ 
possibility is the emergency nature of work during the COVID19 pandemic, which may interfere with sleep. This possibility

Table 4 Univariate and multivariate binary logistic regression analyses for predicting sleep quality, moderate-severe perceive stress, and the combination of both among healthcare workers

\begin{tabular}{|c|c|c|}
\hline Variables in the Equation & OR $(95 \% \mathrm{CI})$ & $P$ value \\
\hline \multicolumn{3}{|l|}{ Poor sleep quality } \\
\hline \multicolumn{3}{|l|}{ Univariate analysis } \\
\hline Age (year) & $1.0(0.9-1.1)$ & 0.5 \\
\hline Female sex & $1.4(0.7-2.6)$ & 0.3 \\
\hline Marital status & $0.3(0.1-1.1)$ & 0.1 \\
\hline Professional background & $0.7(0.5-1.1)$ & 0.1 \\
\hline Type of facility (frontline vs. non-frontline) & $0.9(0.5-1.6)$ & 0.8 \\
\hline \multicolumn{3}{|l|}{ Multivariate analysis } \\
\hline Age (year) & $1.1(1.0-1.1)$ & 0.5 \\
\hline Female sex & $1.4(0.8-2.6)$ & 0.3 \\
\hline Marital status & $0.3(0.1-1.1)$ & 0.08 \\
\hline Professional background & $0.7(0.5-1.1)$ & 0.1 \\
\hline Type of facility (frontline vs. non-frontline) & $0.9(0.5-1.6)$ & 0.8 \\
\hline \multicolumn{3}{|l|}{ Moderate-severe perceived stress } \\
\hline \multicolumn{3}{|l|}{ Univariate analysis } \\
\hline Age (year) & $1.0(0.9-1.1)$ & 0.6 \\
\hline Female sex & $2.1(1.0-4.1)$ & 0.04 \\
\hline Marital status & $1.2(0.4-3.2)$ & 0.8 \\
\hline Professional background & $0.8(0.5-1.2)$ & 0.3 \\
\hline Type of facility (frontline vs. non-frontline) & $1.1(0.6-2.1)$ & 0.8 \\
\hline \multicolumn{3}{|l|}{ Multivariate analysis } \\
\hline Age (year) & $1.0(0.9-1.1)$ & 0.6 \\
\hline Female sex & $2.0(1.1-4.0)$ & 0.04 \\
\hline Marital status & $1.2(0.4-3.2)$ & 0.8 \\
\hline Professional background & $0.8(0.5-1.3)$ & 0.3 \\
\hline Type of facility (frontline vs. non-frontline) & $1.1(0.6-2.2)$ & 0.8 \\
\hline \multicolumn{3}{|c|}{ Combined poor sleep quality and moderate-severe perceived stress ${ }^{\text {a }}$} \\
\hline \multicolumn{3}{|l|}{ Univariate analysis } \\
\hline Age (year) & $1.0(0.9-1.1)$ & 0.3 \\
\hline Female sex & $2.0(1.2-3.5)$ & $0.01 *$ \\
\hline Marital status & $0.6(0.3-1.4)$ & 0.2 \\
\hline Professional background & $0.7(0.5-1.0)$ & $0.05^{*}$ \\
\hline Type of facility (frontline vs. non-frontline) & $1.0(0.6-1.6)$ & 0.8 \\
\hline \multicolumn{3}{|l|}{ Multivariate analysis } \\
\hline Age (year) & $1.0(1.0-1.1)$ & 0.3 \\
\hline Sex & $2.0(1.1-3.5)$ & $0.01 *$ \\
\hline Marital status & $0.6(0.3-1.1)$ & 0.2 \\
\hline Professional background & $0.7(0.5-1.1)$ & $0.05^{*}$ \\
\hline Type of facility (frontline vs. non-frontline) & $0.9(0.6-1.6)$ & 0.8 \\
\hline
\end{tabular}

Postestimation a sensitivity, $88 \%$; specificity, $57 \%$; area under the receiver operating characteristic (ROC) curve, 0.6; Omnibus Tests of Model, $P<0.001$; Hosmer-Lemeshow goodness of fit, $P=0.03$; Nagelkerke R Square, $3.7 \%$ is supported by a recent study, in Saudi Arabia, that showed a high prevalence of poor sleep among emergency room physicians of $83 \%$ using the PSQI [38].

In general, healthcare workers are at an increased risk of work-related stress [41]. In the current study, the mean PSS score was high, with a value of 20.3 . With the added concerns about COVID-19, poor sleep and stress among healthcare workers are expected to increase. This is supported by the finding that severe stress in the present study was more prevalent among FLHCW compared with the NFLHCW. The reported numbers of healthcare victims of SARS-CoV-2 fuel this fear and stress among FLHCW [42]. Another interesting finding in this study is the fact that female sex was a predictor of poor sleep quality and stress. The majority of participates in this study were female nurses. Nurses, in general, as an occupational group, are subjected to multiple stressors [43]. Additionally, women are more likely to complain of sleep problems [36, 37], stress, and neuropsychiatric complaints and somatoform disorders, such as headache, depression, and anxiety $[44,45]$.

The present study demonstrated that sex and being a nonphysician health-allied member were the predictors of the combined poor sleep quality and severe perceived stress. As discussed above, women had been reported to have a higher prevalence of poor sleep quality and stress [46]. Additionally, women tend to have greater bodily attentiveness and awareness of physical symptoms than men [46]. It has been proposed that society, in general, is more receptive to women to express their psychological distress and somatic symptoms [47]. This is another plausible mechanism to explain the increased reporting of poor sleep quality and stress in survey studies.

Poor sleep quality, stress, and mental health problems among healthcare workers could impair their cognitive abilities and their clinical decision-making [48, 49]. Thus, increasing the likelihood of making medical errors that may increase the risk to patients. Moreover, it has been demonstrated that acute stress in disasters could result in a lasting effect on the overall well-being [50]. Therefore, looking after the mental health of healthcare workers during the COVID-19 pandemic should be a crucial public health concern. The development and implementation of mental health assessment, support, and intervention become essential to manage the mental health consequences during the outbreak and after. Even though frontline healthcare workers are categorized as a level one category that is vulnerable to mental health problems, similar to the patient infected with the COVID-19 virus, still, there is a limited number of studies investigating the mental health impacts on these workers [51]. Stress is closely associated with sleep quality and this has been documented by previous research that utilized the PSS and PSQI as research tools [52-54]. Nonetheless, some studies suggested that people who are trained to deal with stress such as (healthcare workers, 
law enforcement officers, etc.) can maintain relatively stable emotional state even under stress, and they may experience fewer episodes of night waking, sleep anxiety, and sleep delay [55]. A recent study from Wuhan, China, that investigated anxiety, stress, self-efficacy, and sleep quality among frontline medical staff treating COVID-19 patients concluded that anxiety, stress, and self-efficacy were mediating variables to social support and sleep quality.

Several factors may contribute to healthcare workers' sleep quality and stress in the working environment, including physical strain caused by the perceived physical isolation (restrictions on interaction with others, even after working hours), the use of protective equipment throughout working hours with the resulting dehydration and exhaustion, the necessity for constant vigilance regarding infection control procedures, long working shifts, and conceivable separation from and concern about family members with fears of infecting family members and beloved ones [21]. A recent review concluded that the severity of stress and lack of sleep was influenced by age, gender, occupation, specialization, prolonged night shifts, type of activities performed, and proximity to COVID-19 patients [56]. These factors need to be addressed in future research as potential confounding factors.

Recommended measures for supporting healthcare providers include the provision of individually psychological support interventions. This includes the provision of training in some aspects that they perceived to be highly important to promote their competence, such as handling emotionally distressed patients, guaranteeing staff daily supplies from protective equipment, meals, and other daily living supplies and provision of accommodation and places for rest and leisure activities. To improve sleep, more focus could be directed toward a healthy diet provided to healthcare workers to be rich with vitamins, minerals, healthy fats (omega-3 fatty acids), prebiotics, and probiotics, with a reduced amount of sugars and energy-dense fast foods [57, 58].

It has been found that the provision of social support during the COVID-19 outbreak has positive impacts on lowering stress responses, anxiety symptoms, along with improving healthcare workers' self-efficacy to enable them to maintain stable emotions and promote self-control during pressure. This indirectly is associated with promoting sleep quality [39].

\section{Strengths and limitations}

There are several strengths to make this study valuable. First, to our knowledge, this is the first study to evaluate the sleep quality of healthcare workers in Bahrain and the Arab World during the pandemic stage of COVID-19 and one of the first in the world. Second, the inclusion of multidisciplinary professionals allowed for comparison between different backgrounds.
However, our study has a few limitations that need to be addressed. First, the reliance on a cross-sectional study does not allow us to analyze sleep and stress over some time. Thus, data from different stages of the pandemic stages are lacking. For example, the alert phase will require significant preparedness efforts, and therefore, stress and sleep disturbances might be different than transition or end stages. Second, we focused on healthcare workers who are involved directly in the provision of care to patients; however, other jobs may expose performers indirectly to COVID-19 patients, such as administrators, communication officers, and logistics. Therefore, these groups must also be studied in future studies. Third, to keep the survey time acceptable, our questionnaires did not address potential confounders, e.g., caffeine or nicotine intakes, diets, medical history, shift work details, and coexisting sleep disorders such as obstructive sleep apnea. Finally, we do not have a control group of participants working outside the healthcare profession.

\section{Conclusion}

The present study showed that about $75 \%$ of healthcare workers had poor sleep quality, $85 \%$ had moderatesevere stress, and $60 \%$ had both poor sleep quality and moderate-severe stress. The differences in PSQI and PSS were not significant between FLHCW and NFLHCW. No association was obtained between perceived stress and sleep quality. Sex and professional background were the most impacting predictors of poor sleep quality and mental health. During pandemics, preventive interventions aiming to maintain the health condition of healthcare workers are warranted.

\section{Compliance with ethical standards}

Conflict of interest The authors declare that they have no conflict of interest.

Ethical approval All procedures performed in studies involving human participants were in accordance with the ethical standards of the institutional and/or national research committee and with the 1964 Helsinki declaration and its later amendments or comparable ethical standards.

Informed consent Informed consent was obtained from all individual participants included in the study.

\section{References}

1. Chen C, Zhou Y, Wang DW (2020) SARS-CoV-2: a potential novel etiology of fulminant myocarditis. Herz 45:1-3. https://doi. org/10.1007/s00059-020-04909-z

2. Somily AM, BaHammam AS (2020) Coronavirus disease-19 (severe acute respiratory syndrome-coronavirus-2) is not just simple 
influenza: what have we learned so far? J Nat Sci Med 3(2):79-82. https://doi.org/10.4103/JNSM.JNSM_22_20

3. Chen N, Zhou M, Dong X, Qu J, Gong F, Han Y, Qiu Y, Wang J, Liu Y, Wei Y, Xia Ja YT, Zhang X, Zhang L (2020) Epidemiological and clinical characteristics of 99 cases of 2019 novel coronavirus pneumonia in Wuhan, China: a descriptive study. Lancet 395(10223):507-513. https://doi.org/10.1016/ S0140-6736(20)30211-7

4. Shereen MA, Khan S, Kazmi A, Bashir N, Siddique R (2020) COVID-19 infection: origin, transmission, and characteristics of human coronaviruses. J Adv Res 24:91-98. https://doi.org/10. 1016/j.jare.2020.03.005

5. World Health Organization. Coronavirus disease (COVID-2019) situation reports, https://www.who.int/emergencies/diseases/ novel-coronavirus-2019/situation-reports

6. World Health Organization. Naming the coronavirus disease (COVID-19) and the virus that causes it, https://www.who.int/ emergencies/diseases/novelcoronavirus-2019/technical-guidance/ naming-the-coronavirus-disease-(covid-2019)-and-the-virus-thatcauses-it

7. Tucci V, Moukaddam N, Meadows J, Shah S, Galwankar SC, Kapur GB (2017) The forgotten plague: psychiatric manifestations of ebola, zika, and emerging infectious diseases. J Global Infect Dis 9(4):151-156. https://doi.org/10.4103/jgid.jgid_66_17

8. Stewart-Ibarra AM, Hargrave A, Diaz A, Kenneson A, Madden D, Romero MM, Molina JP, Macias Saltos D (2017) Psychological distress and zika, dengue and chikungunya symptoms following the 2016 earthquake in Bahía de Caráquez, Ecuador. Int J Environ Res Public Health 14(12). https://doi.org/10.3390/ijerph14121516

9. Wang C, Pan R, Wan X, Tan Y, Xu L, Ho CS, Ho RC (2020) Immediate psychological responses and associated factors during the initial stage of the 2019 coronavirus disease (COVID-19) epidemic among the general population in China. Int $\mathrm{J}$ Environ Res Public Health 17(5). https://doi.org/10.3390/ijerph17051729

10. Levin J (2019) Mental health care for survivors and healthcare workers in the aftermath of an outbreak. Psychiatry Pandemics: 127-141. https://doi.org/10.1007/978-3-030-15346-5_11

11. Cates DS, Gomes PG, Krasilovsky AM (2018) Behavioral health support for patients, families, and healthcare workers. Bioemerg Plan:195-214. https://doi.org/10.1007/978-3-319-77032-1_16

12. Lehmann M, Bruenahl CA, Löwe B, Addo MM, Schmiedel S, Lohse AW, Schramm C (2015) Ebola and psychological stress of health care professionals. Emerg Infect Dis 21(5):913-914. https:// doi.org/10.3201/eid2105.141988

13. Kline C (2013) Sleep quality. In: Gellman MD, Turner JR (eds) Encyclopedia of behavioral medicine. Springer, New York, NY, pp 1811-1813

14. Rosipal R, Lewandowski A, Dorffner G (2013) In search of objective components for sleep quality indexing in normal sleep. Biol Psychol 94(1):210-220. https://doi.org/10.1016/j.biopsycho.2013. 05.014

15. Madrid-Valero JJ, Martínez-Selva JM, Ribeiro do Couto B, Sánchez-Romera JF, Ordoñana JR (2017) Age and gender effects on the prevalence of poor sleep quality in the adult population. Gac Sanit 31(1):18-22. https://doi.org/10.1016/j.gaceta.2016.05.013

16. Qiu D, Yu Y, Li R-Q, Li Y-L, Xiao S-Y (2020) Prevalence of sleep disturbances in Chinese healthcare professionals: a systematic review and meta-analysis. Sleep Med 67:258-266. https://doi.org/10. 1016/j.sleep.2019.01.047

17. Zeng L-N, Yang Y, Wang C, Li X-H, Xiang Y-F, Hall BJ, Ungvari GS, Li C-Y, Chen C, Chen L-G, Cui X-L, An F-R, Xiang Y-T (2019) Prevalence of poor sleep quality in nursing staff: a metaanalysis of observational studies. Behav Sleep Med:1-14. https:// doi.org/10.1080/15402002.2019.1677233
18. AlSaif HI (2018) Prevalence of and risk factors for poor sleep quality among residents in training in KSA. J Taibah Univ Med Sci 14(1):52-59. https://doi.org/10.1016/j.jtumed.2018.11.007

19. Ferri P, Guadi M, Marcheselli L, Balduzzi S, Magnani D, Di Lorenzo R (2016) The impact of shift work on the psychological and physical health of nurses in a general hospital: a comparison between rotating night shifts and day shifts. Risk Manag Healthc Policy 9:203-211. https://doi.org/10.2147/RMHP.S115326

20. Caruso CC (2014) Negative impacts of shiftwork and long work hours. Rehab Nurs 39(1):16-25. https://doi.org/10.1002/rnj.107

21. Koinis A, Giannou V, Drantaki V, Angelaina S, Stratou E, Saridi M (2015) The impact of healthcare workers job environment on their mental-emotional health. Coping Strateg 3(1). https://doi.org/10. 4081/hpr.2015.1984

22. Kumar A, Bhat PS, Ryali S (2018) Study of quality of life among health workers and psychosocial factors influencing it. Ind Psychiatry J 27(1):96-102. https://doi.org/10.4103/ipj.ipj_41_18

23. Mohanty A, Kabi A, Mohanty AP (2019) Health problems in healthcare workers: a review. J Fam Med Prim Care 8(8):2568 2572. https://doi.org/10.4103/jfmpc.jfmpc 43119

24. Jahrami H, Dewald-Kaufmann J, Faris MA-I, AlAnsari AMS, Taha M, AlAnsari N (2019) Prevalence of sleep problems among medical students: a systematic review and meta-analysis. J Public Health. https://doi.org/10.1007/s10389-019-01064-6

25. Medic G, Wille M, Hemels ME (2017) Short- and long-term health consequences of sleep disruption. Nat Sci Sleep 9:151-161. https:// doi.org/10.2147/NSS.S134864

26. Elm EV, Altman DG, Egger M, Pocock SJ, Gøtzsche PC, Vandenbroucke JP (2014) The Strengthening the Reporting of Observational Studies in Epidemiology (STROBE) statement: guidelines for reporting observational studies. Int J Surg 12(12): 1495-1499. https://doi.org/10.1016/j.ijsu.2014.07.013

27. Buysse DJ, Reynolds CF, Monk TH, Berman SR, Kupfer DJ (1989) The Pittsburgh Sleep Quality Index: a new instrument for psychiatric practice and research. Psychiatry Res 28(2):193-213

28. Cohen S (1988) Perceived stress in a probability sample of the United States

29. Manzar MD, BaHammam AS, Hameed UA, Spence DW, PandiPerumal SR, Moscovitch A, Streiner DL (2018) Dimensionality of the Pittsburgh Sleep Quality Index: a systematic review. Health Qual Life Outcomes 16(1):89. https://doi.org/10.1186/s12955018-0915-x

30. Mollayeva T, Thurairajah P, Burton K, Mollayeva S, Shapiro CM, Colantonio A (2016) The Pittsburgh Sleep Quality Index as a screening tool for sleep dysfunction in clinical and non-clinical samples: a systematic review and meta-analysis. Sleep Med Rev 25:52-73. https://doi.org/10.1016/j.smrv.2015.01.009

31. Buysse DJ, Hall ML, Strollo PJ, Kamarck TW, Owens J, Lee L, Reis SE, Matthews KA (2008) Relationships between the Pittsburgh Sleep Quality Index (PSQI), Epworth Sleepiness Scale (ESS), and clinical/polysomnographic measures in a community sample. J Clin Sleep Med 4(6):563-571

32. Jiang JM, Seng EK, Zimmerman ME, Kim M, Lipton RB (2017) Positively worded subscale score of the Perceived Stress Scale is associated with cognitive domain function. J Behav Brain Sci 7(7): 311-324. https://doi.org/10.4236/jbbs.2017.77023

33. Cohen S, Kamarck T, Mermelstein R (1983) A global measure of perceived stress. J Health Soc Behav 24(4):385-396

34. Lee E-H (2012) Review of the psychometric evidence of the perceived stress scale. Asian Nurs Res 6(4):121-127. https://doi.org/ 10.1016/j.anr.2012.08.004

35. Stata MPtE (2019) 16.0. College Station, TX: StataCorp

36. Machi MS, Staum M, Callaway CW, Moore C, Jeong K, Suyama J, Patterson PD, Hostler D (2012) The relationship between shift work, sleep, and cognition in career emergency physicians. Acad 
Emerg Med 19(1):85-91. https://doi.org/10.1111/j.1553-2712. 2011.01254.x

37. Surani AA, Surani A, Zahid S, Ali S, Farhan R, Surani S (2015) To assess sleep quality among Pakistani junior physicians (house officers): a cross-sectional study. Ann Med Health Sci Res 5(5):329333. https://doi.org/10.4103/2141-9248.165246

38. Alhifzi S, Al-Ghonimy A, Al Aboudi M, Al Abdullah R, Olaish A, BaHammam AS (2018) Assessment of sleep quality, daytime sleepiness, and depression among emergency physicians working in shifts. J Nat Sci Med 1:17-21. https://doi.org/10.4103/JNSM. JNSM 8 _ 18

39. Xiao H, Zhang Y, Kong D, Li S, Yang N (2020) The effects of social support on sleep quality of medical staff treating patients with coronavirus disease 2019 (COVID-19) in January and February 2020 in China. Med Sci Monit 26:e923549. https://doi.org/10. 12659/MSM.923549

40. Zhang C, Yang L, Liu S, Ma S, Wang Y, Cai Z, Du H, Li R, Kang L, Su M, Zhang J, Liu Z, Zhang B (2020) Survey of insomnia and related social psychological factors among medical staff involved in the 2019 novel coronavirus disease outbreak. Front Psychiatry 11: 306. https://doi.org/10.3389/fpsyt.2020.00306

41. Merga H, Fufa T (2019) Impacts of working environment and benefits packages on the health professionals' job satisfaction in selected public health facilities in eastern Ethiopia: using principal component analysis. BMC Health Serv Res 19(1):494. https://doi.org/ 10.1186/s12913-019-4317-5

42. Brooks SK, Dunn R, Amlot R, Rubin GJ, Greenberg N (2018) A systematic, thematic review of social and occupational factors associated with psychological outcomes in healthcare employees during an infectious disease outbreak. J Occup Environ Med 60(3): 248-257. https://doi.org/10.1097/JOM.0000000000001235

43. Thompson MR, Schwartz Barcott D (2017) The concept of exposure in environmental health for nursing. J Adv Nurs 73(6):13151330. https://doi.org/10.1111/jan.13246

44. Matud MP, Bethencourt JM, Ibanez I (2015) Gender differences in psychological distress in Spain. Int J Soc Psychiatry 61(6):560 568. https://doi.org/10.1177/0020764014564801

45. Maunder RG, Lancee WJ, Rourke S, Hunter JJ, Goldbloom D, Balderson K, Petryshen P, Steinberg R, Wasylenki D, Koh D, Fones CS (2004) Factors associated with the psychological impact of severe acute respiratory syndrome on nurses and other hospital workers in Toronto. Psychosom Med 66(6):938-942. https://doi. org/10.1097/01.psy.0000145673.84698.18

46. Ter Horst JP, de Kloet ER, Schächinger H, Oitzl MJC, neurobiology m (2012) Relevance of stress and female sex hormones for emotion and cognition. 32(5):725-735

47. Barsky AJ, Peekna HM, Borus JF (2001) Somatic symptom reporting in women and men. J Gen Intern Med 16(4):266-275

48. Panagioti M, Geraghty K, Johnson J, Zhou A, Panagopoulou E, Chew-Graham C, Peters D, Hodkinson A, Riley R, Esmail A (2018) Association between physician burnout and patient safety, professionalism, and patient satisfaction: a systematic review and meta-analysis. JAMA Intern Med 178(10):1317-1330. https://doi. org/10.1001/jamainternmed.2018.3713

49. LeBlanc VR (2009) The effects of acute stress on performance: implications for health professions education. Acad Med 84(10 Supp1):S25-S33. https://doi.org/10.1097/ACM. 0b013e3181b37b8f

50. Mulfinger N, Sander A, Stuber F, Brinster R, Junne F, Limprecht R, Jarczok MN, Seifried-Dubon T, Rieger MA, Zipfel S, Peters M, Stiawa M, Maatouk I, Helass M, Nikendei C, Rothermund E, Hander N, Ziegenhain U, Gulde M, Genrich M, Worringer B, Kullenberg J, Blum K, Suss S, Gesang E, Ruhle S, Contributors of the Sc, Muller A, Schweitzer-Rothers J, Angerer P, Gundel H (2019) Cluster-randomised trial evaluating a complex intervention to improve mental health and well-being of employees working in hospital - a protocol for the SEEGEN trial. BMC Public Health 19(1):1694. https://doi.org/10.1186/s12889-019-7909-4

51. Li W, Yang Y, Liu ZH, Zhao YJ, Zhang Q, Zhang L, Cheung T, Xiang YT Progression of mental health services during the COVID-19 outbreak in China. (1449-2288 (Electronic))

52. Lemma S, Gelaye B, Berhane Y, Worku A, Williams MA (2012) Sleep quality and its psychological correlates among university students in Ethiopia: a cross-sectional study. BMC Psychiatry 12:237237. https://doi.org/10.1186/1471-244X-12-237

53. Rebello CR, Kallingappa PB, Hegde PG (2018) Assessment of perceived stress and association with sleep quality and attributed stressors among 1(st)-year medical students: a cross-sectional study from Karwar, Karnataka, India. Ci Ji Yi Xue Za Zhi 30(4):221-226. https://doi.org/10.4103/tcmj.tcmj_85_17

54. Sano A, Phillips AJ, Yu AZ, McHill AW, Taylor S, Jaques N, Czeisler CA, Klerman EB, Picard RW (2015) Recognizing academic performance, sleep quality, stress level, and mental health using personality traits, wearable sensors and mobile phones. Int Conf Wearable Implant Body Sens Netw. https://doi.org/10.1109/ BSN.2015.7299420

55. Bihlmaier I, Schlarb AA (2016) Self-efficacy and sleep problems. Somnologie 20(4):275-280

56. Bohlken J, Schömig F, Lemke MR, Pumberger M, Riedel-Heller SG (2020) COVID-19 pandemic: stress experience of healthcare workers - a short current review. Psychiatr Prax 47(4):190-197. https://doi.org/10.1055/a-1159-5551

57. Taylor AM, Holscher HD (2020) A review of dietary and microbial connections to depression, anxiety, and stress. Nutr Neurosci. 23(3):237-250

58. Ismaeel A, Weems S, Ellor JW, Crow J, Myers D, Dolan S, Whitacre J, Morissette SBJMBH (2018) Association between negative emotional states and unhealthy nutritional behaviors in soldiers. 6(1):66-74

Publisher's note Springer Nature remains neutral with regard to jurisdictional claims in published maps and institutional affiliations. 